\title{
ILCEA
}

Revue de l'Institut des langues et cultures

d'Europe, Amérique, Afrique, Asie et Australie

$29 \mid 2017$

Les femmes en Russie : parcours, mythes et représentations

\section{Nadejda Mandelstam - gardienne de la mémoire}

НАДЕЖДА МАНДЕЛЬШТАМ — ХРАНИТЕЛЬНИЦА ПАМЯТИ

Natalia Leclerc

\section{OpenEdition}

Journals

Édition électronique

URL : http://journals.openedition.org/ilcea/4277

DOI : 10.4000/ilcea.4277

ISSN : 2101-0609

Éditeur

UGA Éditions/Université Grenoble Alpes

Édition imprimée

ISBN : 978-2-37747-007-5

ISSN : 1639-6073

Référence électronique

Natalia Leclerc, « Nadejda Mandelstam - gardienne de la mémoire », ILCEA [En ligne], 29 | 2017, mis en ligne le 30 juin 2017, consulté le 30 avril 2019. URL : http://journals.openedition.org/ilcea/4277 ; DOI : 10.4000/ilcea.4277

Ce document a été généré automatiquement le 30 avril 2019

(C) ILCEA 


\section{Nadejda Mandelstam - gardienne de la mémoire}

НАДЕЖДА МАНДЕЛЬШТАМ - ХРАНИТЕЛЬНИЦА ПАМЯТИ

Natalia Leclerc

1 «En Russie, on meurt sans rien dire » écrit Nadejda Mandelstam (2013a: 165). C'est ce problème qu'elle affronte dans Contre tout espoir, ses mémoires publiés d'abord en anglais sous le titre Hope against Hope et Hope Abandoned, dans les années 1970, puis en Russie au moment de la perestroïka.

2 Tout au long des mille deux cent cinquante pages de son texte, elle donne des éléments d'explication relatifs à cette incapacité à parler qui frappe ses concitoyens :

À présent, l'inquiétude avait disparu et la peur s'était évanouie. Elles avaient cédé la place à une conscience aiguë du fait que nous étions condamnés, d'où une indifférence physiquement perceptible, palpable, terriblement pesante. Désormais, le temps n'existait plus, il n'y avait plus que des délais avant la réalisation de l'irrévocable qui nous guettait tous, avec notre Europe, avec nos bribes de dernières pensées et de derniers sentiments. (2012:53)

3 C'est le constat de la disparition du temps qui m'intéresse dans ces lignes. Je montrerai que par l'écriture de ses mémoires, Nadejda Mandelstam fait certes œuvre de gardienne de la mémoire, mais aussi du temps, dont elle travaille à restaurer le passage.

4 Ainsi, face à la menace d'un silence complet et accepté à force d'être imposé, silence dont Nadejda Mandelstam se rend compte qu'il est «un véritable crime contre l'espèce humaine» (2012:54), la nécessité de hurler se fait jour, et la métaphore de la bête hurlante parcourt le texte. Ce hurlement bestial est paradoxalement ce qui lui permet de maintenir son humanité. Elle constate d'ailleurs que ses contemporains ont moins de souvenirs que les animaux.

5 Mais elle rencontre aussi la menace de la vanité, comme le suggère la dernière section de l'ensemble, intitulée «Les années de silence ». Nadejda Mandelstam y évoque la possible inutilité du témoignage d'autrui s'il est fragmentaire, et en conclut l'importance pour elle de tout dire, de répéter sans cesse, de viser la totalité, la globalité (2013b:369). Nous 
rencontrerons d'autres facteurs risquant de fragiliser son entreprise et je montrerai comment ils viennent en réalité renforcer l'édifice.

Contre tout espoir est un texte complexe, paradoxal, comme l'indique son titre lui-même. Il rencontre un constat amer et actuel, celui d'une Russie qui n'a pas jugé son passé, qui n'a pas eu son tribunal de Nuremberg, et qui souffre de l'absence d'un travail de mémoire.

\section{Des mémoires indispensables}

\section{Le destin des archives et des écrits}

7 Le travail de Nadejda Mandelstam apparait avant tout comme une nécessité destinée à contrer une fatalité propre à l'époque, et le texte, qui ne suit pas un ordre chronologique, s'ouvre sur ce qui le justifie: la première arrestation de Mandelstam, le problème que représentent ses manuscrits et leur conservation. La venue de la police politique chez Mandelstam s'accompagne d'une confiscation de certains de ses textes, confiscation dont les méthodes évoluent d'ailleurs après 1937 (2012 : 14). Elle constitue l'épisode inaugural de Contre tout espoir, la violence originelle qui motive la rédaction des mémoires.

8 Ainsi, après cette première arrestation, durant laquelle les policiers n'emportent pas tous les textes, parce qu'ils n'ont pas eu l'idée de chercher dans les casseroles ou sur la table, Nadejda Mandelstam et ses amis parviennent à évacuer un certain nombre d'écrits avant une deuxième perquisition, le lendemain. À la fin du premier tome, elle évoque encore les stratégies pour protéger les manuscrits, par exemple celle qui consiste à les disperser chez différents amis (2012:353). La conservation physique des textes, qui deviennent presque des reliques, et le geste de transformer ces textes en archives, en traces mémorielles, a ici une force particulière: il ne s'agit pas de sauver de la destruction ordinaire par disparition ou par usure du temps, mais de la destruction intentionnelle, du saccage de la mémoire et de la confiscation des preuves.

Si Nadejda Mandelstam mémorise les textes de son mari pour éviter leur disparition pure et simple en cas de saisie par le NKVD, la mémoire individuelle n'apparaît pourtant pas comme l'unique solution en ces temps de Terreur et d'extermination: Mandelstam « comprit qu'il était plus facile de préserver un manuscrit qu'un homme, et il cessa de compter uniquement sur sa mémoire" (2012:27). L'homme est périssable - et prématurément - c'est la première de ses finitudes. Nadejda Mandelstam signale aussi la perte définitive des poèmes qu'elle n'a pas réussi à mémoriser, et la perte encore plus grande des poèmes restés à l'état de virtualités jamais actualisées : «[...] ils ont péri par ma faute. Je les regrette, mais je regrette encore plus ceux qui n'ont pas été écrits parce que l'air est venu à manquer. » (2013a: 312) La plus grande violence de l'époque est sans doute d'avoir restreint les horizons, anéanti les possibles, rendu des paroles ou des actes inconcevables, au sens propre du terme.

10 Face à ces limites humaines et circonstancielles, une solution s'impose, la publication d'un texte de mémoires par l'épouse du poète. Mais l'intérêt de Contre tout espoir est que ce travail ne se substitue pas à la mémorisation des poèmes, dont il ne constitue pas le recueil, mais vient la compléter, l'étayer, et possède sa problématique propre, qui relève du témoignage. 


\section{La terreur comme tentative d'extinction de la mémoire}

11 Cette nécessité de témoigner est d'autant plus urgente que l'époque que traverse le couple Mandelstam est vécue comme fondée sur le projet d'annihilation de la mémoire. C'est un processus organisé par le régime, qui veut créer une société nouvelle ( $\mathrm{Chez}$ nous, on encourage la perte de mémoire, et on exécute les gens qui ont gardé la leur " [2013b: 310]) ou qui provoque une accélération incontrôlée et rendant la mémoire impossible ( Je n'étais pas la seule, et tous mes contemporains avaient le sentiment aigu que le temps était devenu fou et fuyait sans laisser la moindre trace dans la mémoire " [2013a: 222]), mais engendrant aussi une réaction de protection des individus (« Pour préserver leurs enfants, les parents les élevaient dans l'ignorance totale » [2013a : 214]).

À la page suivante, on lit cette prophétie qui résonne douloureusement aujourd'hui :

Les hommes dorment parce qu'on les a artificiellement privés de leur mémoire. Il faut qu'ils sachent ce qu'ont vécu les générations de leurs pères et de leurs grandspères, sinon ils entreront sans en être avertis dans un nouvel enchaînement de calamités et s'avéreront totalement sans défense. [...] Il faut rendre aux hommes la mémoire et la peur. (2013a : 215)

- la peur étant ce qui maintient les hommes en vie. La parole prophétique de Nadjeda Mandelstam retentit encore dans le tome III : « Vous descendrez stériles dans vos tombes profondes. » (2013b : 272) On entrevoit ici une des fonctions du témoignage selon Nadejda Mandelstam: celle d'expliquer l'enchaînement des événements, car l'articulation du passé et de l'avenir n'est pas seulement de l'ordre de la succession mais de celui de la causalité, et le témoignage n'est pas seulement un récit didactique mais permet de déduire, de faits advenus, une prophétie pour les générations suivantes.

Mais Nadjeda Mandelstam n'est pas Cassandre et l'entreprise de rédaction des mémoires est également justifiée au plan phénoménologique, dans une longue section intitulée « $\mathrm{La}$ mémoire" (tome II, p. 186 à 200). Nadejda Mandelstam y explique la nécessaire falsification des souvenirs, que celle-ci ait pour origine une volonté politique de récrire l'histoire, le besoin de sauver sa vie («Un seul mot sincère, et - alors comme maintenant - cela m'aurait valu dix ans de bagne... » [2013a : 192]), ou qu'elle relève d'un processus naturel : "La mémoire altère nos souvenirs et ne nous permet pas de prendre conscience ni de l'expérience historique ni de l'expérience individuelle, mais, en même temps, c'est elle qui fait de l'homme un être humain. » (2013a : 194)

Malgré cette conception pessimiste de la nature humaine - relativement dépourvue de conscience - et face à cette falsification quasi inévitable, tout en étant parfois involontaire, Nadejda Mandelstam affirme la nécessité absolue d'une mémoire fiable: «La perte d'une mémoire honnête, non falsifiée, équivaut à la perte du sens du réel. Le présent perd sa signification si les faits peuvent être arrangés et présentés - à soi-même et aux autres - sous n'importe quelle forme. » (2013a: 196) Il est frappant de lire ici une conception du temps et de la mémoire proche de celle de Bergson, que Nadejda Mandelstam cite ailleurs, sur un autre sujet. Bergson refuse une conception spatialisée du temps, mais conçoit une existence dans la durée, où le sujet avance toujours chargé de sa mémoire, et où cette mémoire conditionne et réoriente en permanence son action dans le présent. Il me semble que cette philosophie de la durée est sous-jacente dans la vision de Nadejda Mandelstam. 


\section{Vivre dans le présent et en garder la trace}

15 Ainsi, le devoir de mémoire de Nadejda Mandelstam est un devoir envers elle-même, celui de vivre le temps présent sans qu'il soit distinct du passé, sans que le temps soit perçu comme segmenté, fait de ruptures, vivre dans un temps bergsonien. Mais c'est aussi un devoir envers son mari. Une des particularités d'Ossip Mandelstam, mentionnée au moins une fois dans chacun des trois tomes, est son choix assumé de vivre dans le présent, pour des raisons de contexte historique : «[...] il ne fallait pas regarder vers l'avenir, car il ne promettait rien de bon. [...] Ne rien attendre, et être prêt à tout. Le secret de l'équilibre était là » (2012:78-79), mais aussi pour des raisons de tempérament de poète $(2012: 180-181 ; 2013 a: 218-219 ; 2013 b: 17)$. Ne construisant pas de monument à sa propre mémoire autre que sa poésie - dont la conservation est menacée - Mandelstam induit donc implicitement chez sa femme le devoir de regarder vers l'avenir, de témoigner de son existence.

Ce devoir de prévoir un avenir à Mandelstam est accepté et assumé par sa femme, au nom d'une conception téléologique propre à l'époque : «Mon but était de justifier la vie de Mandelstam en préservant ce qui lui donnait son sens. [...] En gâchant ma vie, on m'a imposé un but. [...] Dans les périodes d'attente, le but remplace le sens. " (2013a:224) En rédigeant ses mémoires, Nadejda Mandelstam réalise ce que Mandelstam lui-même s'était toujours refusé à faire. Elle accomplit ce qu'elle considère être un devoir posthume contre le gré du mort.

La raison pour laquelle ce devoir viole les volontés de Mandelstam, effectuant le contraire de ce qu'il fit de son vivant, est probablement à chercher du côté de la notion d'identité : en travaillant à la subsistance de la vie et de l'œuvre de son mari, Nadejda Mandelstam travaille aussi à la consolidation de la sienne propre. Contre tout espoir n'est pas né d'une injonction extérieure mais d'une nécessité intrinsèque, propre à Nadejda Mandelstam et à sa philosophie du temps.

\section{Le travail d'une mémoire individuelle au service de l'autre et du « nous »}

\section{Je et tu}

18 Le statut de Nadejda Mandelstam dans cette entreprise pourrait sembler ambigu et faire écho à son errance après la mort de Mandelstam. Joseph Brodsky y fait référence lorsqu'il parle, dans la préface (Mandelstam, 2012: III), du « statut de non-personne » de Nadejda Mandelastam. Nadejda Mandelstam ne risque-t-elle pas ainsi d'être réduite au statut de «femme de »? Beth Holmgren rappelle le contexte général :

To some extend these women became writers because they were survivors charged to bear witness. In many cases they were "literary widows" who bravely assumed responsability for their husbands' forbidden archives and biographies. Moreover, in composing autobiographical textes, these women were working in a medium traditionally accessible to Russian women-one that, ideally, afforded them unmediated self-definition and, at the same time, justified what the reading public perceived as the audacity of their authorship because they wrote to commemorate political victims. (1993:2) 

observée chez Mandelstam - comme « quelque chose de voisin de la remémoration de ce qui n'a encore jamais été dit » (2012 : 234). Poésie et mémoire sont deux facultés cousines, ce qui suggère que les rapports entre Nadejda et Ossip constituent une forme particulière de rapport à l'altérité, comme le montre ce très beau passage où Mandelstam exige que Nadejda le tutoie : «Des filles que je tutoie et qui me disent "vous", il y en aura à foison, mais toi, tu es mon "toi"...» (2013a : 169) Ainsi, lorsque Nadejda Mandelstam rapporte les propos d'Akhmatova qui la voit comme le trait d'union entre Ossip et elle-même, qui la considère comme «tout ce qui nous reste d'Ossia » (2013a: 279), il faut voir en Nadejda Mandelstam plus que l'épouse ou le porte-parole de son mari. Elle est son alter ego, elle est sa prose.

\section{Je et nous}

Mais la fonction de son œuvre ne consiste pas seulement à faire exister la poésie de son mari. Elle a une fonction sociale également, à une époque où la structure même de la société est malmenée. 
Tout le début du deuxième volume porte sur le «moi", et sur son anéantissement: «Dans ce magma [celui des arrestations et des déportations], le mot "moi” n'existait plus. C'était presque devenu un mot honteux, un mot interdit. Qui aurait osé parler de son destin, se plaindre de son sort, quand c'était le sort commun?» (2013a:10) Mais par réaction, réhabiliter le «moi » est aussi une manière de lutter contre «l'atrophie de la personnalité » (2013a:13) que le régime met en place. Ainsi, la mémoire de Nadejda Mandelstam permet aussi de faire subsister et même exister le «moi » de Mandelstam à une époque où « la vie n'existait plus » (2013a : 14).

Plus encore, la réhabilitation du « moi » de Mandelstam et la mise en place des conditions de possibilité de sa subsistance sont d'utilité publique, puisque faire exister un «moi», faire exister le «moi », permet aussi de travailler à la construction d'une société où le «nous» ne soit plus un «pluriel purement quantitatif, qui n'est pas cimenté par une signification et un contenu internes» (2013a:35), mais forme un ensemble cohérent: "Le véritable "nous" est intangible, irréfutable et entier. On ne peut pas le briser, le morceler, le détruire. Il reste intangible et entier même lorsque les hommes qui étaient réunis par ce vocable reposent dans leurs tombes. » (2013a : 40)

Dans La Mémoire, l'histoire, l'oubli, Paul Ricœur analyse le rôle du témoignage comme facteur de sûreté dans le lien social. Le témoignage repose sur la confiance dans la parole d'autrui, et justement, celle-ci « renforce [...] la similitude en humanité des membres de la communauté » (Ricœur, 2003 : 207).

\section{Un rôle historique, politique et métaphysique}

\section{Témoigner, connaître, agir}

La fonction de ces mémoires n'a pas seulement à voir avec la persistance de la poésie et $\mathrm{du}$ « moi » de Mandelstam, mais aussi avec la connaissance historique par le biais du témoignage, présenté comme matière première de l'histoire :

Mais comment les historiens feront-ils pour rétablir la vérité si, partout, la moindre parcelle de vérité est noyée dans un amas de monstrueux mensonges? Car ce n'étaient pas des préjugés ou des erreurs dues à l'époque, mais des mensonges conscients et délibérés. (Mandelstam, $2012: 32$ )

Son travail participe donc de la destruction des légendes dont les hommes se souviennent, selon elle, au détriment des événements (2012:211). Elle construit là où le régime a détruit, à moins qu'elle ne détruise les artefacts de l'histoire officielle en relatant sa vision individuelle, singulière.

Ce rétablissement et cette possibilité d'une connaissance historique ne sont pas autotéliques, mais ont une finalité ancrée dans un contexte socio-historique, celle du combat contre le fascisme : «[...] le pire, ce sont les gens à demi instruits, et c'est parmi eux qu'on trouvera toujours un terrain favorable au fascisme, aux formes les plus basses du nationalisme, à la haine des intellectuels.» (2012:437) Le projet de Nadejda Mandelstam est donc motivé au plan épistémologique, mais aussi pragmatique.

Il a enfin une signification morale, puisque la possibilité de présenter des traces mémorielles est aussi un devoir vis-à-vis des générations ultérieures : "Que penseront nos petits-enfants si nous partons tous sans avoir rien dit?» (2012:201) Alexandra Popoff, pour sa part, parle de «mission» : 
For decades, she led a nomadic life, hiding from the authorities and supporting herself with teaching. What kept her alive was her mission to publish Mandelstam's works and tell his story. Nadezhda memorized much of his verse and prose, making her memory an additional storage. After Stalin's death in 1953, she committed to paper Mandelstam's works, along with background information, and began to struggle for posthumous publication. (2012)

\section{Juger le passé}

31 des faits - neutralité qui est d'ailleurs aux antipodes de l'écriture de Nadejda Mandelstam - on passe au jugement. Les responsables de la mort de Mandelstam et de tous ceux de sa génération n'ayant jamais été jugés, le texte de Nadejda Mandelstam fait office de condamnation.

Contrairement aux analyses topiques sur la littérature concentrationnaire et sur l'indicible, Nadejda Mandelstam considère que «[f]ermer les yeux est difficile, cela demande de gros efforts » (2012:74), même si une cécité incurable et une sclérose morale frappent les gens de sa génération (2013a : 228). Il lui faut parler, et loin de représenter un effort, le témoignage et le jugement s'imposent à elle : "On ne peut dissimuler le passé, même quand il n'y a pas de statistiques. Chaque homme exterminé a encore son mot à dire. » (2013a : 178) Parler est une nécessité qui dépasse la simple question morale. Il ne s'agit pas de condamner parce que c'est mal, mais de condamner parce qu'il faut dire ce mal, le formuler, le penser. On s'est interrogé après la Shoah sur la possibilité de penser ce phénomène impensable, et sur la possibilité de continuer à penser tout court. Dire l'époque de la Terreur permet de la penser, et donc de la maintenir vivante en tant que souvenir. Il s'agit de ne pas archiver la Terreur comme un objet d'étude figé, devenu mécanique, mais de la penser comme un organisme potentiellement toujours vivace.

est d'autant plus indispensable que chez ses concitoyens, «[1]a conversation s'était arrêtée, et pour toujours » (2013a:94), de même que la plaisanterie, l'anecdote (2013a: 153), et qu'encore plus en amont, la pensée ellemême s'est arrêtée : «Les mots viennent prendre la relève de la pensée lorsque celle-ci se tarit. » (2013a:17) Les mots dont il est question ici sont mécaniques, ils simulent la pensée. Témoigner comme elle le fait, restaure une parole vive.

Pourtant, la question de la vanité de cette entreprise est posée et rend le travail de Nadejda Mandelstam humain, car fragile autant qu'indispensable. Le pessimisme face à son projet apparaît, me semble-t-il, dans le tome III. On y lit une page très amère qui pourrait invalider ce qu'elle a dit précédemment sur l'aveuglement: "Ma cécité est consciente. Je ne veux pas voir clair.» (2013b : 253) Ces mots ne me semblent pourtant pas être en contradiction avec son désir de garder les yeux ouverts. Au contraire : ils soulignent justement la fragilité de son entreprise, soumise aux défauts de mémoire, mais aussi au découragement, à la perte de la confiance en sa nécessité.

Quelques pages plus haut, elle soulève aussi une question sur le sens de l'histoire: «L'histoire ne se répète pas. Les raisonnements par analogie aboutissent toujours à des conclusions fausses, et surtout dans les cas où l'on utilise des faits falsifiés pour raisonner par analogie. » (2013b : 248) On n'étudie pas l'histoire pour, comme le disent les enfants, éviter de répéter les mêmes erreurs. Mais en même temps, s'il n'y a aucune constance dans les enchaînements causaux, on peut s'interroger sur le risque de la gratuité d'un témoignage historique, qui ne serait tourné que vers le passé. C'est que l'entreprise de 
Nadejda Mandelstam a une portée métaphysique au moins autant qu'historique ou mémorielle.

\section{Restaurer le temps}

"Time is out of joint » disait Hamlet, et l'époque à laquelle a vécu Nadejda Mandelstam a souffert de la même dislocation, dans la mesure où «elle avait faussé la perspective du passé et prédisait l'avenir sur la base de raisonnements faux sur le passé » (Mandelstam, 2013b : 298). Ainsi, le couple Mandelstam se sent étranger de ses voisins temporels, qui n'appréhendent pas l'histoire, et donc l'avenir, de la même façon - surtout si l'on considère la sensibilité de Mandelstam qui, en tant qu'artiste, vit à la fois dans les trois époques et dans son propre temps, ce qui le rend " anachronique ${ }^{1}$ " dans sa manière d'être et dans la perception qu'en ont ses concitoyens.

restaurer le temps, dont leur génération a été privée :

Lorsqu'on vit dans un état de terreur permanente, prêtant l'oreille aux voitures et aux coups de sonnette, on se met à percevoir chaque seconde et chaque minute. Celles-ci s'étendent, prennent du poids et pèsent sur votre poitrine comme du plomb. C'est un état physique et non psychique, particulièrement aigu la nuit. Les minutes se prolongent tandis que les années filent à une vitesse folle, ne laissant derrière elles rien d'autre qu'un vide béant. Les vingt années qui se sont écoulées entre la mort de Mandelstam, en 1938, et la fin des années cinquante me font l'effet d'une boule informe et dénuée de sens, où le temps ne s'écoulait pas mais était agglutiné, puis s'est désagrégé dans le néant. (Mandelstam, 2013a : 304)

Ce n'est donc pas seulement la temporalité historique qui est bouleversée et sciemment déformée, mais aussi la temporalité humaine. Ossip et Nadejda Mandelstam, chacun avec son écriture, tentent de leur redonner une cohérence et une lisibilité. Ainsi, pour Brodsky, les mémoires de Nadejda Mandelstam «sont plus qu'un témoignage sur son temps, c'est une vision de l'Histoire à la lumière de la conscience et de la culture " (Mandelstam, 2012: VIII), tandis que Nadejda souligne que pour son mari, la poésie est une « victoire sur le temps » (2013b: 18).

En conclusion, j'aimerais revenir à la figure féminine, que je n'ai abordée que de biais tout au long de cette présentation. La différence entre la vie des hommes et celle des femmes sous la Terreur stalinienne revient régulièrement sous la plume de Nadejda Mandelstam. Dans les faits et dans les statistiques, les femmes «couraient quand même moins de risques que les hommes» (Mandelstam, 2012:48), pour ce qui est des arrestations. Par ailleurs, elle constate que les femmes sont physiquement plus endurantes que les hommes (2012 : 394). C'est peut-être cette résistance qui leur donne ce rôle de pilier, dans le foyer, et dans l'histoire: "Ce sont les femmes, véritables gardiennes du foyer domestique, qui entretiennent les lueurs d'espoir avec une force démoniaque » (2012:42) ou "[l]es temps difficiles firent apparaître le rôle organisateur de la femme qui faisait semblant d'être une dame, fragile et délicate, mais était en réalité une bâtisseuse et le principal support du foyer » (2013a : 131).

Mais qu'elle soit gardienne du foyer ne suffit pas encore à faire de Nadejda Mandelstam une gardienne de la mémoire. C'est Mandelstam lui-même qui décèle cette potentialité chez son épouse qui apparait presque comme une sur-femme. Chassant une voyante, il souligne ce qu'il estime être son omniscience : «Qu'as-tu besoin d'interroger l'avenir ? Tu sais déjà tout. » (2013a : 78) 
41 Ainsi, on pourrait dire que Nadejda Mandelstam dépasse la différence établie par Ricœur entre le juge et l'historien. Le philosophe, après avoir souligné les points communs entre ces deux fonctions - le fait que tous deux s'appuient sur le témoignage, cherchent des preuves, le fait qu'un procès suive un déroulement analogue à une investigation historiographique - montre que la finalité de leur réflexion les différencie fondamentalement : là où la sentence du juge conduit à la restauration d'un ordre social ébranlé, le travail de l'historien mène à une perpétuelle réécriture. Là où le juge s'adresse à des individus, l'historien parle à toute une population. Où situer Nadejda Mandelstam? Elle échappe à cette confrontation entre le juge et l'historien, comme elle échappe au processus mis en place dans l'ensemble de l'ouvrage de Ricœur, qui mène à un pardon apaisé. Nadejda Mandelstam est à la fois juge et historien, parce qu'elle amorce le travail de jugement des coupables de la Terreur des années 1930 et parce qu'elle s'appuie pour cela sur des traces. Mais si l'on en croit Mandelstam, elle n'est ni juge ni historien, elle qui «sai[t] tout", et n'a pas besoin d'investiguer ni de juger. En revanche, par son omniscience, elle est la gardienne d'un matériau qui, déjà utilisé par l'historien, pourra, espérons-le, être utilisé par le juge pour condamner les crimes encore aujourd'hui impunis.

\section{BIBLIOGRAPHIE}

Holmgren Beth (1993), Women's Works in Stalin's Time. On Lidiia Chukovskaia and Nadezhda

Mandelstam, Bloomington et Indianapolis : Indiana University Press.

MANDELSTAM Nadejda (2012) [1972], Contre tout espoir, Souvenirs I [1970], traduction Maya

Minoustchine, Paris : Gallimard, coll. « Tel ».

MANDELSTAm Nadejda (2013a) [1974], Contre tout espoir, Souvenirs II [1973], traduction Maya

Minoustchine, Paris : Gallimard, coll. « Tel ».

MANDELSTAM Nadejda (2013b) [1975], Contre tout espoir, Souvenirs III [1973], traduction Maya

Minoustchine, Paris : Gallimard, coll. « Tel ».

Popoff Alexandra (2012), The Wives. The Women Behind Russia's Literary Giants, New York : Pegasus Books.

RICœUR Paul (2003), La Mémoire, l'histoire, l'oubli, Paris : Seuil, coll. « Essais ».

\section{NOTES}

1. Mandelstam (2013b: 12, 20): «Mandelstam était considéré comme un véritable anachronisme. » 


\section{RÉSUMÉS}

Nadejda Mandelstam a sauvegardé de multiples façons les poèmes de son mari, mort en déportation en 1938. Ce travail ne portera pas uniquement sur la transmission mais aussi sur la préservation de la mémoire, à une époque et sous un régime où tout est fait pour annihiler le passé, la notion d'héritage et même de temps. Nadjeda Mandelstam en gardienne permet également, grâce aux traces qu'elle a laissées dans son témoignage, de juger un passé totalitaire qui n'a pas encore été jugé.

НАДЕЖДА МАНДЕЛЬШТАМ, ИСПОЛЬЗУЯ САМЫЕ РАЗНООБРАЗНЫЕ МЕТОДЫ, СМОГЛА СОХРАНИТЬ СТИХИ СВОЕГО МУЖА, ОСИПА МАНДЕЛЬШТАМА, УМЕРШЕГО В ССЫЛКЕ В 1938 ГОДУ. В ДАННОЙ РАБОТЕ ИССЛЕДУЕТСЯ НЕ ТОЛЬКО ПЕРЕДАЧА ПАМЯТИ, НО И ВОЗМОЖНОСТЬ ЕЁ СОХРАНЕНИЯ В ТОТ ПЕРИОД И ПРИ ТОМ РЕЖИМЕ, КОГДА ПРИЛАГАЮТСЯ ВСЕ УСИЛИЯ ДЛЯ ТОГО, ЧТОБЫ УНИЧТОЖИТЬ ПРОШЛОЕ, И САМИ ПОНЯТИЯ НАСЛЕДСТВА И ДАЖЕ ВРЕМЕНИ. БЛАГОДАРЯ ТЕМ СЛЕДАМ, КОТОРЫЕ ОНА ОСТАВИЛА В СВОЁМ СВИДЕТЕЛЬСТВЕ, НАДЕЖДА МАНДЕЛЬШТАМ, В КАЧЕСТВЕ ХРАНИТЕЛЬНИЦЫ ПАМЯТИ, ДАЁТ ТАКЖЕ ВОЗМОЖНОСТЬ СУДИТЬ О ТОТАЛИТАРНОМ ПРОШЛОМ, КОТОРОЕ НЕ БЫЛО ЕЩЁ ОСУЖДЕНО.

INDEX

motsclesru ТЕРРОР, ПАМЯТЬ, ПОЭЗИЯ, ТЕМПОРАЛЬНОСТЬ, СУЖДЕНИЕ

Mots-clés : terreur, mémoire, poésie, temporalité, jugement

\section{AUTEUR}

NATALIA LECLERC

Université de Bretagne occidentale 